\title{
Teledermatología en Atención Primaria
}

\section{Alba Fernández Gómez ${ }^{\mathrm{a}}$, Marcos Clavero Adell ${ }^{\mathrm{a}}$, Irene García Osés ${ }^{\mathrm{b}}$, José M.․ Arnal Alonsoc, Carmen Puig Garcíac ${ }^{c}$, Francisco Javier García Latasa de Aranibar ${ }^{d}$}

Publicado en Internet:

14-junio-2017

Alba Fernández Gómez: albafgo90@gmail.com
a⿳MIR-Pediatría. Servicio de Pediatría. Hospital Universitario Miguel Servet. Zaragoza. España • b Pediatra. CS de Valtierra. Navarra. España • 'Pediatra. CS Actur Norte. Zaragoza. España • 'Servicio de Dermatología. Hospital Royo Villanova. Zaragoza. España.
Palabras clave:

- Dermatología

- Atención Primaria

- Telemedicina

- Consulta a distancia
Las nuevas tecnologías son una herramienta cada vez más utilizada en el desarrollo de la asistencia clínica. La teledermatología pretende establecer una comunicación rápida y eficaz entre el facultativo de Atención Primaria y el especialista en Dermatología. Se expone una muestra del proyecto de calidad llevado a cabo entre los pediatras de un centro de salud del sector 1 de Zaragoza y un especialista en Dermatología del Hospital Royo Villanova. Se describe el cuadro clínico y las imágenes realizadas en la consulta de Atención Primaria de cinco pacientes en edad pediátrica, así como la respuesta por parte del especialista. La teledermatología es un proyecto con buena aceptación por parte de pacientes y profesionales; disminuye listas de espera, evita derivaciones innecesarias y fomenta el aprendizaje por parte del pediatra.

\section{Teledermatology in Primary Care}

Key words:

- Dermatology

- Primary Health care

- Telemedicine

- Remote consultation
New technologies are an increasingly used tool in the development of clinical care. Teledermatology aims to establish a fast and efficient communication between the primary care physician and the specialist in dermatology. We exposed a sample of the quality project carried out between the pediatricians at a health centre in sector 1 in Zaragoza and a specialist in dermatology of the Hospital Royo Villanova. Is describes the picture clinical and the images made in the consultation of attention primary of 5 patients in age Pediatric, as well as the response from the specialist. Teledermatology is a project with good acceptance by patients and professionals; decreases waiting lists, avoids unnecessary referrals and encourages learning by the pediatrician.

\section{INTRODUCCIÓN}

Las alteraciones cutáneas en Pediatría suponen un motivo de consulta muy frecuente. El uso de nuevas tecnologías permite la comunicación entre el especialista y el médico de Atención Primaria, mejorando la práctica clínica a nivel de las consultas en el centro de salud. La teledermatología consiste en establecer un sistema de comunicación continuo entre ambos facultativos.

Por estos motivos, se lleva a cabo el plan de calidad que pretende dar respuesta a la población pediátrica

Cómo citar este artículo: Fernández Gómez A, Clavero Adell M, García Osés I, Arnal Alonso JM, Puig García C, García Latasa de Aranibar FJ. Teledermatología en Atención Primaria. Rev Pediatr Aten Primaria. 2017;19:171-5. 
que acuda a consulta con una patología dermatológica, y en la que se presentan dudas diagnósticoterapéuticas.

El proyecto se presentó y fue seleccionado dentro del Programa de Apoyo a las Iniciativas de Mejora de la Calidad en Salud del Gobierno de Aragón en la convocatoria de 2011, y se ha mantenido en las siguientes convocatorias.

Dicho proyecto consiste en el envío por parte del pediatra de Atención Primaria de un correo electrónico a los profesionales del Servicio de Dermatología del sector 1 de Zaragoza, añadiendo una breve historia clínica junto con las imágenes de las lesiones de cada paciente, el mismo día en que acude a la consulta de Atención Primaria. Las imágenes son tomadas con cámara digital previo consentimiento verbal de los padres, y en caso de ser mayores de 12 años, también de los propios pacientes, manteniendo la confidencialidad. Los dermatólogos realizan una valoración clínica del caso y responden por correo electrónico con las recomendaciones oportunas.

En los años 2015 y 2016 se han realizado 12 y 10 consultas respectivamente. En el año 2015, la media de contestación fue de cuatro días y en 2016 de tres días, siendo el tiempo máximo de derivación de 10 y 11 días respectivamente. Como consecuencia de la buena aceptación de los pacientes y profesionales, se decide incluir una encuesta de satisfacción a rellenar por los padres con el objetivo de evaluar este servicio. A continuación, se presentan algunos de los casos más representativos.

\section{SERIE DE CASOS}

\section{Caso 1}

Niña de cinco años que presenta una única lesión maculopapulosa, eritematosa, de carácter circinado, con bordes ligeramente sobreelevados, de unos $5 \mathrm{~cm}$ de diámetro, localizada en la palma de mano (Fig. 1). Se trata de una lesión pruriginosa sin mejoría tras tratamiento corticoideo tópico.

Desde el Servicio de Dermatología se descarta la escabiosis por la forma y distribución de las lesiones y
Figura 1. Lesión maculopapulosa, eritematosa, de carácter circinado, con bordes ligeramente sobreelevados, de unos $5 \mathrm{~cm}$ de diámetro

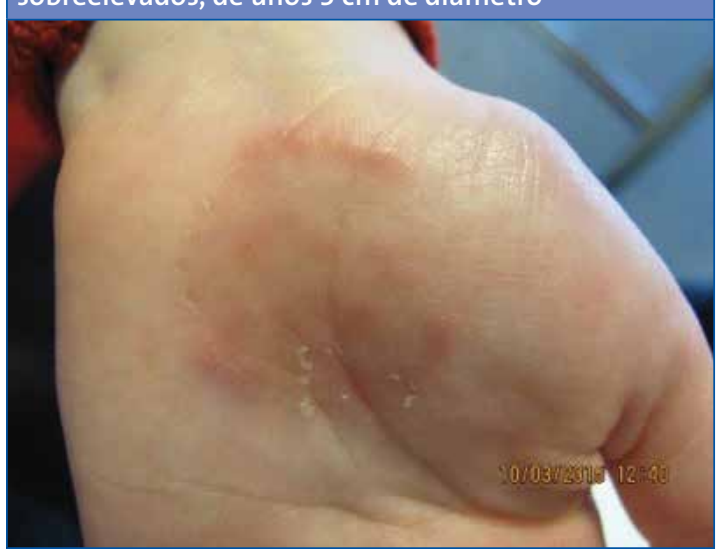

el granuloma anular por presentar descamación. Finalmente se llega al diagnóstico de una tiña incógnita anteriormente tratada con corticoides, que disminuyen la inflamación y dificultan el diagnóstico, manteniendo la lesión activa. Se pauta tratamiento antimicótico durante seis semanas.

\section{Caso 2}

Paciente de 12 años de edad que acude a consulta por presentar lesiones en el tórax de $0,5 \times 0,5 \mathrm{~cm}$, eritematosas, ligeramente descamativas, no pruriginosas (Fig. 2). En el brazo presenta otra lesión de características similares, de mayor tamaño, también asintomática (Fig. 3). Ante la sospecha de

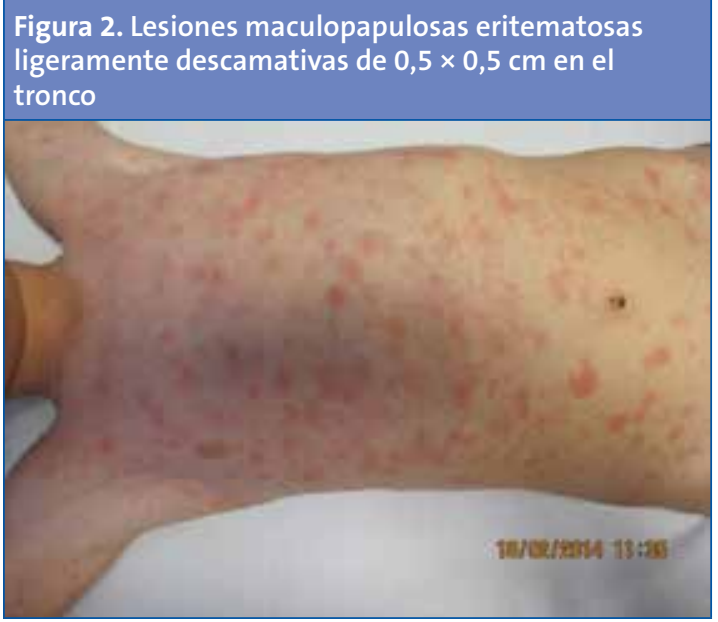




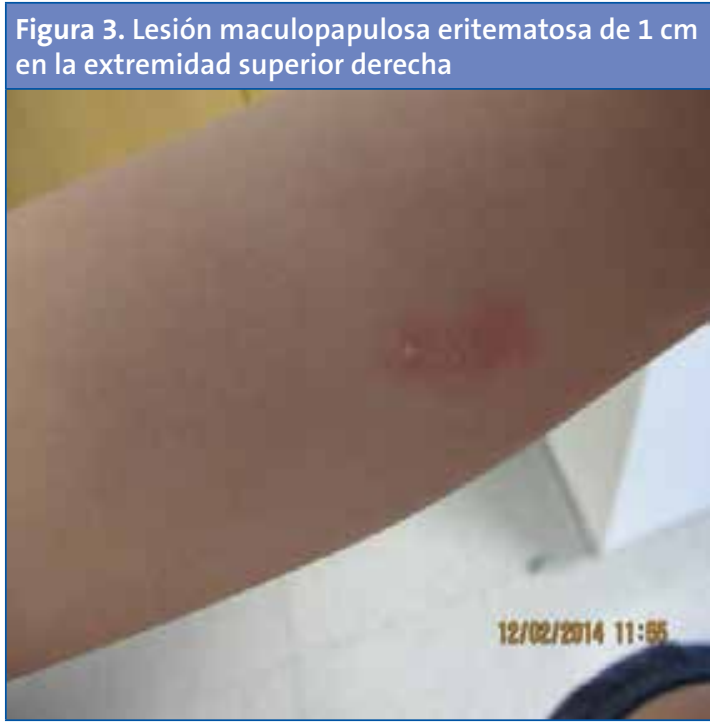

pitiriasis rosada, no se pauta tratamiento y se espera para ver la evolución del cuadro.

A la semana acude de nuevo a consulta, presentando un aumento del número de lesiones, distribuidas por el tronco, el abdomen, el dorso y la región cervical, y respetando la cara, el cuero cabelludo y las extremidades inferiores. Al igual que la semana anterior, las lesiones son asintomáticas.

Dado la cantidad de lesiones, se decide consultar vía telemática a Dermatología, quienes emiten como diagnóstico más probable una pitiriasis rosada, debido a la probable lesión heraldo del brazo, la descamación periférica en algunas lesiones y la distribución.

Aconsejan no tratar y nos recuerdan que el cuadro puede durar un total de 6-8 semanas.

\section{Caso 3}

Niño de nueve años que acude a consulta con una lesión eritematosa circinada, con bordes sobreelevados, eritematosos, notablemente más activos que el centro de la lesión, de unos $10 \mathrm{~cm}$, situada en el muslo derecho (Fig. 4). Tratada con hidratación desaparece en unas semanas. Sin embargo, a los meses presenta lesiones similares de menor tamaño dispersas en tórax, cuello y muslo.
Figura 4. Lesión eritematosa circinada, con bordes sobreelevados, eritematosos, notablemente más activos que el centro de la lesión, de unos $10 \mathrm{~cm}$

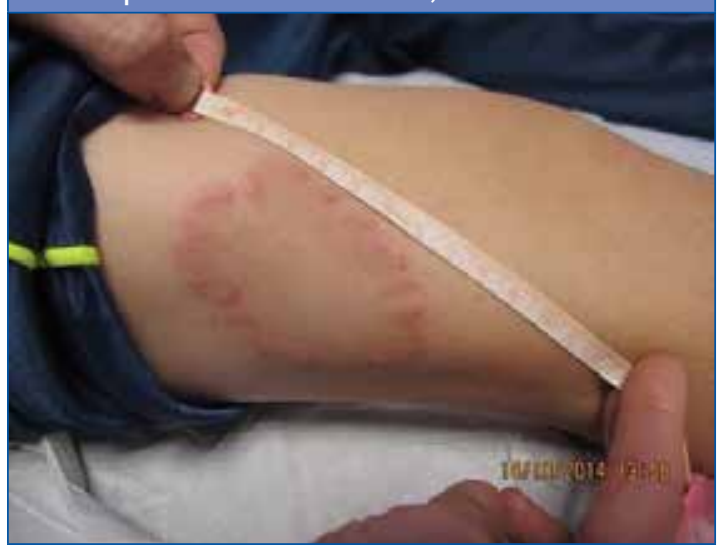

Se realiza un cultivo de las lesiones, con resultado negativo. Dado el prurito intenso y la resolución espontánea con hidratación, el dermatólogo responde que la etiología más probable es eccema atópico. Por ello, aconseja tratamiento con corticoide tópico.

\section{Caso 4}

Niña de cinco años que presenta una lesión quística sobreelevada azulada de $1 \mathrm{~cm}$ de diámetro en la raíz de cuero cabelludo (Fig. 5), con un aumento de tamaño en las sucesivas visitas. Según el especialista, la lesión sugiere un pilomatricoma o epitelioma calcificante de Malherbe (puede ser azulado

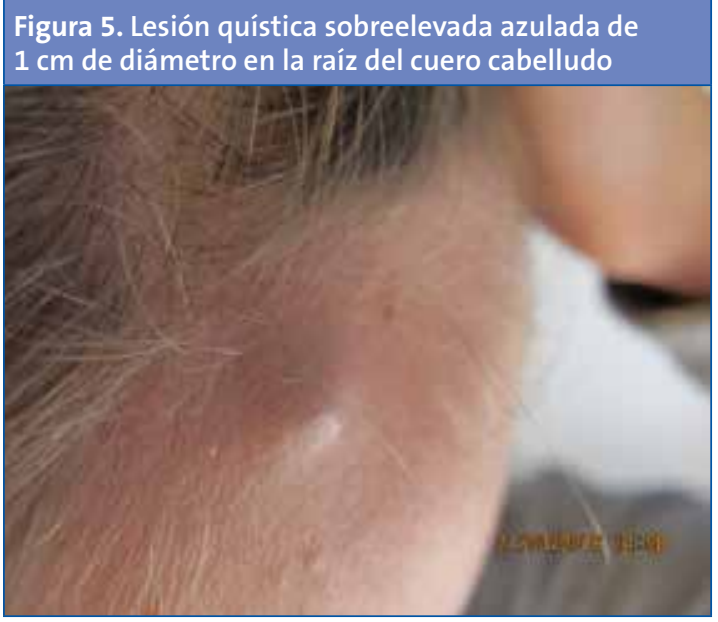


por su componente cálcico). Recomienda la extirpación quirúrgica como actuación diagnósticoterapéutica.

\section{Caso 5}

Paciente de dos años, afecto de neurofibromatosis, que presenta una lesión lineal consistente en la agrupación de pequeñas pápulas pálidas sobreelevadas sin signos inflamatorios en el abdomen de unos $7 \mathrm{~cm}$ de largo (Fig. 6). La lesión presenta fenómeno de Koebner. Tras la interconsulta telemática, el diagnóstico más probable es de molluscum contagiosum, sin precisar tratamiento.

\section{DISCUSIÓN}

La presencia de las nuevas tecnologías en el campo de la medicina ha supuesto un auténtico avance. Siendo la telemedicina una disciplina en expansión, lo es más en el campo de la Dermatología dada la importancia que tiene en esta especialidad la imagen. Así lo demuestra el aumento de utilización de esta forma de comunicación entre profesionales desde que se iniciara en 1995 $5^{1,2}$.

La mayoría de los estudios realizados sobre esta forma de trabajo se centran en población adulta. En edad pediátrica existen algunos recientemente publicados $^{3-5}$. En las revisiones llevadas a cabo, la teledermatología se muestra como un método eficiente y efectivo, reduciendo el tiempo de desplazamiento de los pacientes, el tiempo de espera, la frecuentación de las consultas de especialistas y evitando la derivación de casos que pueden ser solucionados de forma telemática ${ }^{6}$.

La opinión de los profesionales se ha valorado mediante encuestas y es positiva en general, si bien es cierto que el perfil de paciente óptimo sería aquel con clínica dermatológica frecuente, o bien aquellos
Figura 6. Lesión lineal consistente en la agrupación de pequeñas pápulas pálidas sobreelevadas en el abdomen, de unos $7 \mathrm{~cm}$ de largo

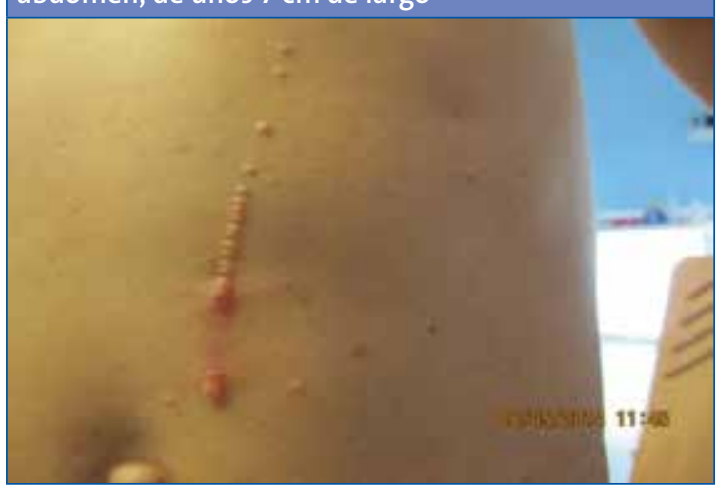

en seguimiento diagnóstico ya establecido. En casos complejos existe preocupación acerca del buen manejo del paciente ${ }^{7}$. La necesidad de derivación al especialista tras la consulta por vía telemática suele ser por la mala calidad de las imágenes o bien aquellos casos con dermatosis más complejas $^{8}$.

Por otro lado, la valoración que el paciente tiene acerca de esta forma de atención sanitaria es de gran importancia, por ello en los próximos meses se incluirá en nuestro plan de mejora una encuesta para los padres de los pacientes, con el objetivo de mejorar la atención.

Aunque cada vez más extendida, todavía está por definir el papel de la teledermatología dentro del sistema sanitario de nuestro país. En otros países sí que se incluye en programas desarrollados dentro de los sistemas sanitarios, como en el caso de algunos estados de EE. UU. y de los Países Bajos ${ }^{6}$.

\section{CONFLICTO DE INTERESES}

Los autores declaran no presentar conflictos de intereses en relación con la preparación y publicación de este artículo. 


\section{BIBLIOGRAFİA}

1. Fieleke DR, Edison K, Dyer JA. Pediatric teledermatology a survey of current use. Pediatr Dermatol. 2008; 25:158-62.

2. Batalla A, Suh-Oh H, Abalde T, Salgado-Boquete $L$, de la Torre C. Teledermatología en edad pediátrica. Observaciones en la práctica clínica diaria. An Pediatr (Barc). 2016;84:324-30.

3. Chen TS, Goldyne ME, Mathes EF, Frieden IJ, Gilliam AE. Pediatric teledermatology: Observations based on 429 consults. J Am Acad Dermatol. 2010;62:61-6.

4. Martinez-García S, del Boz-González J, MartínGonzález T, Samaniego-González E, Crespo-Erchiga V. Teledermatología. Revisión de 917 teleconsultas. Actas Dermosifiliogr. 2007;98:318-24.
5. Vaño-Galván S, Hidalgo A, Aguayo-Leiva I, Gil-Mosquera M, Rios-Buceta L, Plana MN, et al. Teledermatología diferida: análisis de validez en una serie de 2000 observaciones. Actas Dermosifiliogr. 2011;102:277-83.

6. Tensen E, van der Heijden JP, Jaspers MW, Witkamp L. Two Decades of Teledermalogy: Current Status and Integration in National Healthcare Systems. Curr Derm Rep. 2016;5:96-104.

7. Fogel AL, Teng JM. Pediatric teledermatology: a survey of usage, perspectives, and practice. Pediatr Dermatol. 2015;32:363-8.

8. Paradela-De-La-Morena S, Fernandez-Torres R, MartínezGómez W, Fonseca-Capdevila E. Teledermatology: diagnostic reliability in 383 children. Eur J Dermatol. 2015; 25:563-9. 